\title{
Revisiting the Shape of the Yield Curve: The Effect of Interest Rate Volatility ${ }^{1}$
}

\author{
Charlotte Christiansen ${ }^{2}$ \\ The Aarhus School of Business, Denmark \\ cha@asb.dk \\ and \\ Jesper Lund \\ Nykredit Bank, Denmark \\ JL1204@worldonline.dk
}

First draft: February 2001

Current draft: March 13, 2002

\footnotetext{
${ }^{1}$ The authors gratefully acknowledge comments and suggestions from Jesper Rangvid, the participants at the Centre for Analytical Finance members' meeting 2001, Arne Ryde Workshop in Empirical Finance at Lund University, and the European Financial Management Association 2001 Conference. Of course, the authors are responsible for any remaining errors.

${ }^{2}$ Corresponding author: Charlotte Christiansen, Department of Finance, The Aarhus School of Business, Fuglesangs Alle 4, DK-8210 Aarhus V, Denmark. Phone: +458948 6691, fax: +45 8615 1943, email: cha@asb.dk, homepage: www.asb.dk/ cha.
} 


\title{
Revisiting the Shape of the Yield Curve: The Effect of Interest Rate Volatility
}

\begin{abstract}
:
This paper examines the relationship between interest-rate volatility and the shape of the yield curve. The yield curve is parsimoniously described by its level, slope, and curvature. The level, the slope and the curvature are analyzed within a trivariate heteroskedastic model, where the conditional short-rate volatility is included in the mean specification. The slope and the curvature depend positively and significantly on the short-rate volatility. The effect of the interest rate volatility is more pronounced for the curvature than for the slope. Differences between subperiods are explored, as are differences across the maturity spectrum.
\end{abstract}

Keywords: Multivariate GARCH-M, Short-Rate Volatility, Yield Curve Curvature, Yield Curve Shape, Yield Curve Slope

JEL Classifications: C13; C32; G10 


\section{Introduction}

There is a vast and rapidly growing literature on modeling interest rate volatility. Most papers in this area focus on pricing fixed income derivatives relative to the current yield curve. As established by Heath, Jarrow and Morton (1992), arbitrage-free prices of fixed income derivatives only depend on the forward-rate volatility structure, whereas the drift, or conditional mean, of forward rates is irrelevant. Another part of the literature considers the relationship between interest-rate volatility and the shape of the yield curve, in particular the slope and curvature of the yield curve. Our paper contributes to this strand of the literature. Our analysis takes an empirical route using multivariate GARCHin-mean (generalized autoregressive conditional heteroskedasticity) models.

A widely cited study by Litterman and Scheinkman (1991) has found that the term structure of interest rates is driven by a small number of common factors. Using principal components analysis, Litterman and Scheinkman (1991) show that about $95 \%$ of the variation in the yield curve for US treasury bonds is driven by three factors, and they interpret these factors as the level, steepness and curvature of the yield curve. In the last decade, a number of studies have estimated theoretical term-structure models from time series data, in particular multi-factor versions of the Cox, Ingersoll and Ross (1985) model. Most of these papers strongly reject one-factors models and conclude that at least two or three factors are needed to capture the yield curve dynamics adequately, see, e.g., Chen and Scott (1993), Dai and Singleton (2000), and De Jong (2000).

The paper by Litterman, Scheinkman and Weiss (1991) was among the first to suggest that there is a direct link between interest rate volatility and the shape of the yield curve. Litterman et al. (1991) show that there is a high correlation between implied volatility from bond options and yield spreads on certain butterfly combinations. Using a simple binomial term-structure model, they trace the relationship between volatility and the butterfly spread to the convexity of the bond prices (as functions of yields). Andersen and Lund (1997) estimate a three-factor term-structure model with stochastic volatility, and they use the model to show that volatility changes can explain changes in the curvature of the yield curve.

In an empirical study, Brown and Schaefer (2000) document that the term structure of long-term forward rates is downward sloping. Specifically, they consider the spread between the 25-year and the 15-year instantaneous forward rates. A theoretical explanation for this result is the downward convexity bias, which also explains the relationship between volatility and the curvature of the yield curve. In a one-factor model, Brown 
and Schaefer (1994) show that the second derivative of the forward rate with respect to a kind of duration function is equal to the short-rate volatility. Brown and Schaefer (2000) use this result to calculate an implied volatility from the shape of the yield curve.

Engle and $\mathrm{Ng}$ (1993) describe the excess returns of Treasury bills above the risk-free short rate using the one-factor model of Engle, $\mathrm{Ng}$ and Rothschild (1990) in which the common factor is an equally weighted portfolio of the bills in the sample, and the portfolio is assumed to evolve according to a GARCH-in-mean specification. Changes in the yield curve are driven by changes in the expectation about the future short rates and changes in the term premia. The APT model provides a linear relationship between the term premia and the volatility of the common factor. The authors show that their model is able to produce yield curves of the commonly observed shapes. When volatility is high, the yield curve is likely to be upward sloping, and when the volatility is low, the shape of the yield curve is determined mostly by the expectations component since risk premia are virtually absent.

In the present paper, we use a multivariate GARCH-in-mean model to explain the relationship between volatility and the shape of the yield curve. Our paper is related to Engle and Ng (1993), but there are some important differences. First, we model interest rates for selected maturities directly, rather than through risk-premia for expected bond returns. In part, this is because we do not rely on a formal pricing model such as the APT model. Second, we study the effect of volatility changes on the slope as well as the curvature of the yield curve. This is done simultaneously with a trivariate GARCH-inmean specification. Finally, the data used by Engle and Ng (1993) consist of Treasury bills, which means that the largest maturity is one year. We use zero-coupon interest rates for maturities up to 15 years. Hence, although there are several similarities with respect to the econometric methodology, we should expect to obtain different empirical results than Engle and $\mathrm{Ng}$ (1993).

The main empirical findings of the paper can be summarized as follows: Firstly, changes in the level of the yield curve are independent of the short-rate volatility. Secondly, the yield curve is steeper, the greater the short-rate volatility. Thirdly, the yield curve tends to become more concave when short-rate volatility increases. The short-rate volatility plays a more prominent role for the curvature than for the slope of the yield curve. Finally, the results are robust and reasonably well-behaved with respect to changes in the maturities of the zero-coupon bonds under investigation.

The outline of the remaining part of the paper is as follows. In Section 2 the data are introduced after which the econometric framework is discussed in Section 3. The 
empirical analysis is provided in Section 4, and in Section 5 we offer some conclusions.

\section{Description of Yield Curve Data}

To explore the shape of the yield curve, we use the continuously compounded zero-coupon yields covering the short and the medium maturity spectrum: 3 month, 1 year, 2 year, 3 year, 5 year, 7 year, 10 year, and 15 year. We analyze the period from January 1970 through December 1998, where the following non-overlapping subperiods have our special attention: January 1970 through December 1978, January 1979 through December 1982, and January 1983 through December 1998. Thus, we break the sample into the period before, during, and after the so-called "monetary experiment" of the Federal Reserve. These four samples provide us with a total of $1515,468,209$, and 838 weekly observations, respectively. The yields are obtained on Wednesdays when possible, and otherwise on Thursdays or Tuesdays (in that order). The yield curve data come from the Bliss term structures and are constructed using the smoothed Fama and Bliss (1987) method. ${ }^{1}$

Figure 1 displays the surface plot of the weekly yields. The plot represents simultaneously the cross section point of view (the yield on a given date as a function of the time to maturity) as well as the time series point of view (the yield of a given maturity as a function of the calendar day). The yield curve attains its sample maximum level around the monetary experiment. Hardly any of the yield curves are inverse (downward sloping), and, in general, they assume familiar shapes. This is perhaps even more evident from Figure 2 which shows the average yield curves for each of the 27 years that the sample covers. $^{2}$

Table 1 contains summary statistics for the entire period as well as the three subperiods. Overall, the mean yield is increasing with the time to maturity, though the relationship is reversed for the period around the monetary experiment. The standard deviations of the first differences are generally decreasing in maturity, corresponding to a decreasing volatility structure. Furthermore, the average yields are much higher during the monetary experiment; actually, around twice the size in the other periods. It is also the case that the volatilities are much higher during the monetary experiment than in the other two subperiods where the volatilities are of about the same magnitude. In all cases, the Jarque-Bera test documents that the yield series are not normally distributed.

We are not able to reject the null hypotheses of a unit root for all yield series for

\footnotetext{
${ }^{1}$ The yield curve data were obtained from Robert R. Bliss, Federal Reserve Bank of Atlanta.

${ }^{2}$ The surface plot and the average yield curves do not include the 15 year yields.
} 
all time periods, neither using the Dickey-Fuller test nor the Phillips-Perron specification (the alternative hypothesis includes an intercept, a time trend, and a 5th order lag structure). Tests for unit roots are known to have low power, and thus, the results are also consistent with near unit-root behavior. Nonetheless, the Dickey-Fuller tests suggest that we should analyze the yields using first differences instead of levels. Finally, there are strong indications that yield innovations are conditionally heteroskedastic because the squared changes in yields are significantly autocorrelated.

\section{Econometric Framework}

In order to investigate the effect of volatility on the yield curve, we have to decide on the model framework to be applied. The road first forks when we choose between a theoretical multi-factor term-structure model and a descriptive econometric model. For the present purpose, we find the latter approach more convenient.

\subsection{Model specification}

Litterman and Scheinkman (1991) argue that the yield curve can be described by three factors: Level, slope, curvature. Instead of applying a full fledged multivariate model for a large number of points on the term structure (maturities), we exploit the level-slopecurvature representation to reduce the complexity of the empirical analysis.

The curvature of the yield curve can be parsimoniously described by a so-called butterfly portfolio, cf. Litterman et al. (1991), Jones (1991), and De Jong (2000). A butterfly portfolio consists of a long position in an intermediate maturity bond (the body of the butterfly or, alternatively, the bullet part of the butterfly) and a short position of two bonds whose maturities straddle the first bond (the wings of the butterfly or, alternatively, the barbell part of butterfly). For simplicity, we first consider a butterfly where the maturity difference between the body and both wings is identical and where the weight of the body is twice that of each of the wings. Thus, the butterfly spread, $c_{t}$ is given as

$$
c_{t}=y_{t}^{\text {body }}-\frac{1}{2}\left(y_{t}^{\text {leftwing }}+y_{t}^{\text {rightwing }}\right) \text {. }
$$

To understand how the butterfly captures the curvature of the yield curve, let us take a look at Figure 3. We note that when the butterfly spread is positive (by far the most common case), the yield curve is concave and also, the greater the butterfly spread the more concave is the yield curve. This applies both for a normal and for an inverted yield curve. Equivalently, a negative butterfly spread indicates a convex yield curve. 
In most cases, the butterfly position is constructed so that the duration is identical for the bullet side and the barbell side. Hence, the yield spread of the butterfly is given as follows:

$$
c_{t}=y_{2 t}-\left(w y_{1 t}+(1-w) y_{3 t}\right)
$$

where $w$ is chosen such that duration of the barbell position matches that of the bullet position. In equation (2), the subscript $i$ on $y_{i t}$ refers to the bond maturity (with 1 being the shortest maturity, and so forth).

Since the duration of the butterfly is zero, the value of the position is unaffected by small changes in the yield curve (note that a parallel change is implicitly understood in this context). However, the barbell position has a greater bond convexity than the bullet side, which means that the butterfly position has negative convexity. This follows because the bond convexity is proportional to the square of the bond duration. Consequently, a duration-neutral position with negative convexity is adversely affected by "large" (in principle, non-infinitesimal) changes in the yield curve of either sign. The disadvantage of negative convexity is greater when volatility is high, as large changes in the yield curves are more likely.

However, for a market equilibrium to exist, there must also be a benefit of negative convexity. The position with negative convexity earns money when the yield curve stays constant, or more precisely, when sufficiently small changes occur during the given horizon. ${ }^{3}$ When the yield curve does not change, the realized return of the butterfly position is approximately given by the yield spread in (2). Jones (1991) refers to this yield spread as the "horizontal yield give-up" or the cost of convexity. Through these arguments, we have established a theoretical relationship between the curvature of the yield curve, as measured by the butterfly spread, and the level of interest-rate volatility. Specifically, holding other factors constants, an increase in interest-rate volatility should be accompanied by a higher butterfly spread.

The slope of the yield curve is measured by the difference between two yields, a longmaturity yield minus a shorter-maturity yield. Conceptually, the slope of the yield curve depends on expected future changes in the short rate as well as the risk premium for the long-maturity bond, which is usually called the term premium. Engle et al. (1990) use the APT model coupled with a factor-ARCH specification to obtain a linear relationship between term premia and interest-rate volatility. The term-structure models developed by Longstaff and Schwartz (1992) and Fong and Vasicek (1991) provide theoretical support

\footnotetext{
${ }^{3}$ What constitutes "sufficiently small" changes depends on the time horizon, the negative convexity of the butterfly position, and the dynamic properties of the yield curve.
} 
for this specification. Since the average yield curve has a positive slope, cf. Section 2, we should generally expect to obtain a positive relationship between the slope of the yield curve and interest rate volatility (as do Engle et al. (1990)). At the same time, it is important to realize that the slope dynamics are likely to be dominated by the expected future changes in the short rate, so controlling for this factor in the empirical analysis is extremely important. This point is particularly relevant when the yield curve is downward sloping.

In the empirical analysis, the slope of the yield curve is defined by

$$
s_{t}=y_{3 t}-y_{1 t}
$$

Alternatively, we could have defined the slope as, say, $y_{2 t}-y_{1 t}$. However, in terms of keeping the correlation between the slope and the butterfly spread at a reasonable level, the definition in equation (3) is the most appropriate choice. ${ }^{4}$

The 3 month yield is applied as a proxy for the instantaneous short term interest rate, and in our analysis it corresponds to the level of the term structure. Specifically, the first differences of the short rate are analyzed, $\Delta l_{t}=y_{1 t}-y_{1 t-1}$, cf. the unit root discussion in the previous section. Interest rates of shorter maturity are available form our data source, but Duffee (1996) has documented that the idiosyncratic variation in yields with maturities below 3 month makes them poor proxies for the short rate.

\subsection{Properties of the Slope and the Butterfly Spread}

Initially, we limit ourselves to one triplet of maturities: The short rate, $y_{1}$, is the 3 month yield, the medium rate, $y_{2}$, is the 3 year yield, and the long yield, $y_{3}$, is the 10 year yield.

Let us take at brief look at the sample characteristics of the first differences of the 3 month yield, the 10 year $/ 3$ month slope, and the 3 year $/ 3$ month $/ 10$ year butterfly spread defined above in (3) and (2). Table 2 discloses huge differences across time periods. There are by far more negative slopes and butterfly spreads around the monetary experiment (1979-82) than in the two periods surrounding it. In fact, more than $50 \%$ of the days in the 1979-82 subsample have a negative slope and curvature. After 1983 there are hardly any convex or downward sloping yield curves (less than $7 \%$ of the observations). A intermediate situation applies for the first period, but for the full sample (1970-98) an

\footnotetext{
${ }^{4}$ The main empirical analysis in Section 4.2 is based on the (3 month, 3 year, 10 year) maturity triplet. For the 1983-98 period, the correlation between the 3 year $/ 3$ month slope and the 3 year $/ 3$ month/10 year butterfly spread is 0.95 . With the 10 year $/ 3$ month slope, we reduce this correlation to 0.75 .
} 
inverted or a convex yield curve is a fairly rare event. ${ }^{5}$ The standard deviations of the slope and the butterfly spread are large relatively to the means. The standard deviation of the first difference of the short rate is greatest in the 1979-82 period and smallest in the 1983-98 period. The time series of the $\Delta$ level, the squared $\Delta$ level, slope, the squared slope, the butterfly spread and the squared spread are significantly autocorrelated, indicating conditional heteroskedasticity.

The correlations between the $\Delta$ level, slope and curvature have become weaker during the sample period, cf. Table 2. The correlation between the $\Delta$ level and the slope is negative in all three periods. A negative relationship also applies for the $\Delta$ level and the curvature in the first two periods. However, in the most recent subperiod, this correlation is positive. The slope and the curvature are positively correlated in all three periods. Thus, once again the appropriateness of splitting the sample up into sub periods is illustrated. In contrast to the yield series, a unit root is rejected for the $\Delta$ level, the slope, and the butterfly spread series.

\subsection{The Model}

The empirical model for the level-slope-curvature representation of the yield curve combines a vector autoregression (VAR) for the mean equation with a multivariate GARCHin-mean model for the error term. The VAR specification is introduced in order to accommodate the high degree of autocorrelation present in the short-rate changes, the slope, and the butterfly spread. The lag order $p$ of the $\operatorname{VAR}(p)$ specification is determined based on the Schwartz information criterion in a homoskedastic VAR specification (i.e., ignoring the GARCH aspect). This suggests a lag structure of 4 .

The vector $x_{t}$ stacks the first difference of the short rate (level), the slope and the butterfly spread (curvature), that is $x_{t}^{\prime} \equiv\left(\Delta l_{t} s_{t} c_{t}\right)$. Using this notation, the conditional mean equation is given by:

$$
x_{t}=\Phi_{0}+\Phi_{1} x_{t-1}+\Phi_{2} x_{t-2}+\Phi_{3} x_{t-3}+\Phi_{4} x_{t-4}+\gamma \sqrt{h_{1 t}}+\varepsilon_{t},
$$

where $\Phi_{0}$ and $\gamma$ are $(3 \times 1)$ parameter vectors, and $\Phi_{1}, \Phi_{2}, \Phi_{3}$, and $\Phi_{4}$ are $(3 \times 3)$ parameter

\footnotetext{
${ }^{5}$ The joint distribution of the slope and the curvature can be categorized as follows: Slope, curvature $>0$ : $76.4 \%$, slope $<0$, curvature $>0: 7.0 \%$, slope $>0$, curvature $<0: 4.8 \%$, and slope,curvature $<0$ : $11.8 \%$.
} 
matrices. The vector of error terms $\varepsilon_{t}$ has mean zero and conditional covariance matrix:

$$
H_{t} \equiv\left(\begin{array}{ccc}
h_{1 t} & h_{12 t} & h_{13 t} \\
h_{12 t} & h_{2 t} & h_{13 t} \\
h_{31 t} & h_{32 t} & h_{3 t}
\end{array}\right) .
$$

Thus, $\sqrt{h_{1 t}}$ in (4) is the conditional volatility of the short rate. The conditional means depend on the level of the conditional short-rate volatility. As argued in Section 3.1, a positive relationship is expected to prevail both for the slope and for the curvature.

The conditional covariance matrix evolves according to the CCORR specification proposed by Bollerslev (1990), with the individual conditional variance processes following a $\operatorname{GARCH}(1,1)$ specification:

$$
h_{i t}=\omega_{i}+\alpha_{i} \varepsilon_{i t-1}^{2}+\beta_{i} h_{i t-1}
$$

for $i=1,2,3$. For the CCORR specification, the conditional correlation coefficient is assumed to be constant.

$$
h_{i j t}=\rho_{i j} \sqrt{h_{i t} h_{j t}}
$$

for $i, j=1,2,3$ and $i \neq j$. To ensure that the conditional variance is strictly positive the following parameter restrictions must be fulfilled: $\omega_{i}>0, \alpha_{i}, \beta_{i} \geq 0$, for $i=1,2,3$. The persistence of shocks to the conditional variance $\left(h_{t i}\right)$ is measured by $\alpha_{i}+\beta_{i}$, and in order for the conditional variance process to be covariance stationary, the sum of $\alpha_{i}$ and $\beta_{i}$ must be less than one.

In the following, the trivariate model specified by equations (4)-(7) is referred to as the VAR-CCORR-M model. The model parameters are estimated simultaneously using Quasi Maximum Likelihood (QML) with a Gaussian likelihood function and applying robust standard errors, cf. Bollerslev and Wooldridge (1992). ${ }^{6}$

\section{Empirical Results}

\subsection{Model Estimates}

The VAR-CCORR-M model is estimated for the following time periods: 1970-78, 197982, 1983-98, and 1970-98, cf. Table 3. The VAR parameters from the conditional mean

\footnotetext{
${ }^{6}$ The estimation is conducted in GAUSS using the GAUSS module Constrained Maximum Likelihood. A combination of the Berndt-Hall-Hall-Hausman and the Newton-Raphson numerical maximization algorithm is applied. Starting values are set to their unconditional values and the likelihood function is maximized disregarding the first 10 observations.
} 
equation are not reported in the table because, in the present context, they are not interesting in themselves.

Some common features for the volatility-in-mean parameter, $\gamma$, are found across the sample periods. First, with one exception, the volatility of the short-rate changes is not significant in explaining the short-rate changes. Second, the slope depends positively on the short-rate volatility, i.e. $\gamma_{2}$ is positive. ${ }^{7}$ Third, the same applies for the curvature, i.e. $\gamma_{3}$ is positive.

The dependence of the conditional mean of the slope and the curvature on the shortrate volatility is quite different across time periods. In the period before the monetary experiment, 1970-78, both the slope and the curvature of the yield curve depend significantly on the short-rate volatility (though marginally at the $10 \%$ significance level). During the monetary experiment, 1979-82, more than half the observations are for inverted and convex yield curves, which may explain why the volatility dependence is clearly insignificant for both the slope and curvature. In the most recent time period, 1983-98, the volatility of the short-rate changes affect the slope and the curvature significantly ( $p$-values for a two-sided $t$-test equal $4.4 \%$ and $0.2 \%$, respectively). In the 1970-98 period taken as a whole, neither the slope nor the curvature volatility dependence is significant at any reasonable level. The main reason for this is the anomalous period around the monetary experiment. ${ }^{8}$ Once again, this shows why it is important to conduct subperiod analysis. We will return to the quantitative volatility effects shortly, cf. Section 4.2.

The estimated VAR-CCORR-M model documents vast differences in the level of the conditional volatility of the short-rate changes across the various time periods under consideration. This is graphically illustrated in Figure 4 which shows the estimated conditional volatility obtained from the 1970-98 period. It can also be illustrated by observing the three samples separately where the mean (standard deviation) for the conditional volatility is $0.20(0.07), 0.60(0.14)$, and $0.13(0.07)$, respectively. For the entire period the comparable figure is $0.21(0.17)$.

Finally, we examine the suitability of the model by looking for signs of misspecification

\footnotetext{
${ }^{7} \gamma_{i}$ denotes the $i$ th element of the $\gamma$ vector for $i=1,2,3$.

${ }^{8}$ We repeat the analysis for the $1970-98$ period, including dummy variables, so that the volatility-inmean parameters $\gamma_{2}$, and $\gamma_{3}$ can take on different values in the 1979-82 period. In contrast to the results without the 1979-82 dummies (cf. Table 3, Panel A), $\gamma_{2}$ and $\gamma_{3}$ are now jointly significant at the $10 \%$ level. Thus, these findings suggest that the insignificance of the volatility-in-mean parameters is caused by the anomalous period around the monetary experiment. Moreover, the results for the 1970-98 period are robust to the choice of the short rate: The analysis is repeated applying the 1 year yield as the short term interest rate. The results (not shown) are similar to those obtained using the 3 month yield: $\hat{\gamma}^{\prime}=(-0.045,0.028,0.022)$.
} 
in the three series of studentized residuals for each of the four sample periods. If the model is correctly specified, the studentized residuals have mean zero, unit variance, are serially uncorrelated, and the squared studentized residuals are uncorrelated as are the cross multiplied standardized residuals. The only sign of misspecification is that one of the series of the cross multiplied studentized residuals is serially correlated. ${ }^{9}$

\subsection{Volatility Effects on the Yield Curve Shape}

As the effect of short-rate volatility on the yield-curve shape appears to be weak during the monetary experiment, we will leave out this period from the following analysis. During the 1979-82 period, the yield curve often takes on atypical shapes, especially strongly downward sloping and convex shapes. It is quite plausible that this prevents us from finding a relationship between the yield-curve shape and the short-rate volatility that is sufficiently robust across subsamples.

A positive relation between the slope and the yield-curve volatility is expected to prevail. The empirical results support this conjecture, at least if we look at the subperiods before and after the monetary experiment, where the yield curve tends to be steeper, the greater the interest-rate volatility. Moreover, the dependence of the slope on the conditional volatility is somewhat stronger in the first subperiod, 1970-78, than the third subperiod, $1983-98 ; \hat{\gamma}_{2}$ equals 0.33 and 0.21 , respectively. The fact that the yield curve slope depends on the short-rate volatility is interesting from the perspective of the ongoing research on the expectations hypothesis puzzle; see, e.g., Campbell and Shiller (1991), Bekaert, Hodrick and Marshall (1997) and Dai and Singleton (2002). It is possible that risk premia depending on the yield-curve volatility could help resolve the expectation hypothesis puzzle(s).

From an economic point of view, the quantitative effect of the conditional volatility on the yield curve slope is also of interest. For the 1970-78 period, the slope is made up of $33 \%$ of the current volatility $\left(\hat{\gamma}_{2}=0.33\right)$. Hence, for the $1970-78$ period, the average volatility contribution to the slope amounts to $0.066\left(\hat{\gamma}_{2} \sqrt{\bar{h}_{1}}\right)$, and the minimum and the maximum contributions are 0.040 and 0.131 , respectively, $\left(\hat{\gamma}_{2} \min \left[\sqrt{h}_{1 t}\right]\right.$ and $\left(\hat{\gamma}_{2} \max \left[\sqrt{h}_{1 t}\right]\right)$. These numbers must be compared to an average slope of 1.204 (standard deviation of 1.195). For the recent period, 1983-98, the slope is made up of $21 \%$ of the current volatility.

\footnotetext{
${ }^{9}$ We also test whether the lag structure for the VAR specification can be shortened, i.e. we test the null hypothesis of $\operatorname{VAR}(p)$ against the alternative hypothesis of $\operatorname{VAR}(4)$ for $p=3,2,1$. Except for the 1970-78 period, we reject the null hypotheses, indicating that the chosen lag specification is appropriate. For the 1970-78 period, a VAR(2) appears to be preferable.
} 
Therefore, the volatility contribution to the slope is 0.026 on average, with a minimum and maximum of 0.012 and 0.152 , respectively. Again, it makes sense to compare to the average slope of 2.003 (standard deviation of 1.084).

We expect the yield curve to be more concave, the greater the short-rate volatility, cf. the discussion in Section 3.1. The empirical relationship $\left(\gamma_{3}\right.$-estimate) has the right sign in all subperiods, and it is statistically significant before and after the monetary experiment. The conditional mean of the butterfly spread is comprised of about $24 \%$ of the current conditional volatility of the short-rate changes in both subperiods.

In order to assess the economic importance of short-rate volatility, we describe its contribution to the conditional mean of the butterfly spread when volatility equals its minimum, average, and maximum values: Before the monetary experiment, we get 0.025 , 0.042 , and 0.083 , respectively. ${ }^{10}$ These figures should be compared with a mean butterfly spread of 0.486 (standard deviation of 0.538). For the most recent subperiod, 1983-98, the minimum, average, and maximum volatility contribution to the curvature amounts to $0.014,0.030$, and 0.171 , respectively. In this period the average butterfly spread is somewhat higher; 0.665 (and its standard deviation lower; 0.423 ).

A priori, we expect the curvature to be more exposed to term structure volatility changes than the slope, because Litterman and Scheinkman (1991) find that the curvature factor is much stronger correlated with the term-structure volatility than with the slope factor. At first, this may seem to be at odds with our results since the point estimates of $\gamma_{2}$ and $\gamma_{3}$ are close to each other (and the difference is not statistically significant). However, when we take into account the relative sizes of the slope and the butterfly spread (curvature), it is actually the case that volatility plays a more prominent role for the curvature than for the slope of the yield curve.

\subsection{Maturity Effects}

Thus far, we have only used a single triplet of maturities to construct the level, slope, and curvature variables, namely $\left\{y_{1}, y_{2}, y_{3}\right\}=\{3$ month, 3 year, 10 year $\}$. In this section, we estimate the VAR-CCORR-M model for different maturity triplets. The main purpose of this analysis is to investigate whether there are systematic variations in the volatility dependence across the maturity spectrum that can be explained, or rationalized, by economic arguments, such as the convexity of the different butterfly positions. We consider this investigation to be an important robustness check of our empirical results since,

\footnotetext{
${ }^{10}$ The minimum, average, and maximum volatility contributions to the curvature are calculated equivalently to the those for the slope: $\hat{\gamma}_{3} \min \left[\sqrt{h}_{1 t}\right], \hat{\gamma}_{3} \sqrt{\bar{h}_{1}}$, and $\hat{\gamma}_{3} \max \left[\sqrt{h}_{1 t}\right]$.
} 
admittedly, the VAR-CCORR-M model does not rest on a firm theoretical foundation (such as a formal term-structure model with stochastic volatility). As in the previous section, the volatility dependence of the yield curve is measured by the volatility-in-mean parameters $\gamma_{2}$ and $\gamma_{3}$ (for the slope and curvature, respectively). We restrict our attention to the most recent subperiod, 1983-98, i.e. after the monetary experiment, because the previous section has convinced us that this period (possibly along with the 1970-78 period) is the most interesting subperiod in our sample. Moreover, in order to conserve space, we keep the short rate, $y_{1 t}$, fixed at the 3 -month interest rate, so we only consider the effect of varying the maturities for the medium-term yield, $y_{2 t}$, and the long-term yield, $y_{3 t}$.

In theoretical term-structure models, changes in volatility have two distinct effects on the yield curve [see Andersen and Lund (1997) for an in-depth discussion]. If risk premia depend on the stochastic volatility factor, an increase in volatility causes longterm interest rates to increase, so that investors are compensated for the additional risk. The second effect is the downward convexity bias which works in the opposite direction, that is, it pushes long-term yields lower when volatility increases. In most cases, the magnitude of the risk-premium effect is greater than the downward convexity bias, and we should therefore expect to find a positive relationship between the slope of the yield curve and interest-rate volatility. The evidence in Section 4.1 (see Table 3) is consistent with this claim since the estimates of $\gamma_{2}$ are positive for all subperiods.

In Table 4, we keep the short and medium maturities fixed at, respectively, 3 months and 3 years (the same values as in Section 4.1), and we vary the maturity for $y_{3 t}$ from 5 years to 15 years; see panels A through D in Table 4 . Since we define the yield curve slope as $s_{t}=y_{3 t}-y_{1 t}$, we obtain estimates of $\gamma_{2}$ for different maturities of the long bond. As in Table 3, all estimates of $\gamma_{2}$ are positive in Table 4. Quite interestingly, the estimate of $\gamma_{2}$ decreases monotonically as the maturity of the long bond increases. There are at least two plausible explanations for this result. First, the relative importance of the downward convexity bias increases with the maturity of the long bond. Second, we cannot rule out that the risk premium associated with the stochastic volatility factor will start to decrease with maturity at some point. Without a formal term-structure model with stochastic volatility [such as the models put forth by Fong and Vasicek (1991) or Chen (1996)], we can only speculate as to which explanation is the most likely one. In any case, it should be stressed that the differences in the point estimates of $\gamma_{2}$ in Table 4 are smaller than the typical standard error of $\gamma_{2}$.

The economic explanation for the positive relationship between interest-rate volatility 
and the butterfly spread is discussed above in Section 3.1. Basically, part of the butterfly spread can be viewed as a compensation for the disadvantage of negative convexity (similar, in spirit, to the "time decay" for options). In the VAR-CCORR-M model, this effect is captured by the term $\gamma_{3} \sqrt{h_{1 t}}$ in the conditional mean for $c_{t}$, the butterfly spread. By the same line of reasoning as in Section 3.1, we strongly expect estimates of the coefficient $\gamma_{3}$ to be positively related to the negative convexity of the butterfly position.

The convexity of a zero-coupon bond with $\tau$ years to maturity is $\tau^{2}$. Hence, if $\tau_{i}$ denotes the maturity for the yield $y_{i}$, the convexity of the butterfly position (with the opposite sign) is given by:

$$
\mathrm{cvx}=\frac{\tau_{3}-\tau_{2}}{\tau_{3}-\tau_{1}} \tau_{1}^{2}+\frac{\tau_{2}-\tau_{1}}{\tau_{3}-\tau_{1}} \tau_{3}^{2}-\tau_{2}^{2}
$$

since $w=\left(\tau_{3}-\tau_{2}\right) /\left(\tau_{3}-\tau_{1}\right)$ in order for the butterfly position to have zero duration. For fixed values of $\tau_{1}$ and $\tau_{2}$, the convexity (8) increases monotonically in $\tau_{3}$. If $\tau_{1}$ and $\tau_{3}$ are fixed, the maximum value of $(8)$ is obtained when $\tau_{2}=\left(\tau_{1}+\tau_{3}\right) / 2$, that is when we have the greatest dispersion of maturities between the bullet and barbell parts of the butterfly position.

In Table 4, the estimates of $\gamma_{3}$ (the volatility-in-mean coefficient for $c_{t}$ ) increase as we move from Panel A to Panel D. The negative convexity of the butterfly positions also increase from Panel A to Panel D since $\tau_{3}$ increases while $\tau_{1}$ and $\tau_{2}$ are kept fixed. Hence, the evidence in Table 4 supports our contention that the relationship between volatility and the butterfly spread (curvature) represents a compensation for negative convexity.

To shed further light on this issue, we move to Table 5, where the maturities $\tau_{1}$ and $\tau_{3}$ are kept fixed at 3 months and 10 years (as in Table 3 ), and $\tau_{2}$ is varied from 2 to 7 years. Using equation (8), the negative convexity of the four butterfly positions are 14.0 (Panel A), 19.3 (Panel B), 23.8 (Panel C), and 20.3 (Panel D). With respect to the volatility-curvature relationship, the evidence in Table 5 is less clear-cut than in Table 4. All estimates of $\gamma_{3}$ are positive and significant at conventional levels, but the greatest values of $\gamma_{3}$ are found in Panels $\mathrm{A}$ and $\mathrm{B}$, and not in Panel $\mathrm{C}$, as we would have expected from the differences in convexity of the four butterfly positions. However, this could be explained by the effect of volatility on bond risk premia and hence the yield-curve slope. To see this, note that the butterfly spread in equation (2) can be rewritten as

$$
c_{t}=\left(y_{2 t}-y_{1 t}\right)-(1-w)\left(y_{3 t}-y_{1 t}\right)
$$

that is as the difference between two yield-curve slopes. There is some, albeit inconclusive, evidence in Table 4 to suggest that a stochastic volatility factor mainly affects bond risk 
premia for short to medium-term maturities. Since the maturity of $y_{2}$ increases as we move from Panel A to Panel D, the volatility-slope relationship could explain the apparently inconsistent pattern of the $\gamma_{3}$-estimates that we see in Table 5 .

In summary, overall, the dependence of the slope and the curvature of the yield curve on the short-rate volatility behaves as expected when the maturities of the bonds used to represent the slope and the curvature of the yield curve are varied. Specifically, we find further support for a systematic relationship between volatility on one hand and yield-curve slope and curvature on the other.

\section{Conclusion}

In this paper, we investigate the empirical relationship between interest-rate volatility and the shape of the yield curve. The dynamics of the interest-rate volatility is modeled with a multivariate GARCH process, and the shape of the yield curve is represented through its slope and curvature, the latter being defined as a so-called butterfly spread. In terms of methodology, our study provides a framework where the correlation between the short-rate volatility on one hand, and the slope and curvature of the yield curve on the other, has been specified ex ante. This compares favorable to the ex post analysis offered by most other studies.

The full sample spans the period 1970-1998, but due to a structural break during the monetary experiment (1979-82), we find it more convenient to consider the subsamples 1970-78 and 1983-98. For these two subperiods, the GARCH-in-mean coefficients capturing the relationship between short-rate volatility and slope and curvature are statistically significant. Moreover, both coefficients are positive, as expected. The most plausible economic reasons for this result are, respectively, a term premium depending on a stochastic volatility factor (slope) and a compensation for the negative convexity of a duration-neutral butterfly position (curvature). Furthermore, we investigate whether the volatility-slope and volatility-curvature relationships are robust across the maturity spectrum. These results indicate that short-rate volatility has the strongest effect on term premia for short and medium-term maturities. We find some variation in the volatility-curvature relationship, but most of this can be rationalized by the convexity of

the different butterfly positions (and, hence, is in accordance with our a priori economic reasoning).

The positive interpretation of our empirical results is that we have uncovered a systematic relationship between, especially, interest-rate volatility and yield-curve curvature, 
as well as between volatility and the slope of the yield curve. Both features are present in multi-factor term-structure models with stochastic volatility, such as those of Fong and Vasicek (1991) and Chen (1996). In other words, our results suggest that the stochastic volatility factor of these models will be useful in explaining changes in the yield curve itself (and not just higher-order moments).

However, we should also acknowledge that volatility typically plays a relatively minor role in explaining either the yield-curve slope or curvature, at least in comparison with the other driving forces for the term structure (such as expected changes in the short rate which feeds into the yield curve slope, cf. the expectations hypothesis). The relatively high standard errors for the GARCH-in-mean coefficients in Tables $3-5$ are also symptomatic of this. In a recent paper, Collin-Dufresne and Goldstein (2002) develop a term-structure model with "unspanned" stochastic volatility, i.e. a volatility factor which does not affect the yield curve itself, and the authors provide some empirical support for this type of specification. Since there are substantial differences with respect to the econometric methodology between their study and ours, we shall not attempt to compare the empirical results. However, if a large part of the time-varying volatility is unspanned (in the CollinDufresne and Goldstein (2002) sense), it is not surprising that volatility tends to play a relatively minor role in explaining the yield-curve shape in our paper. 


\section{References}

Andersen, T. G. and Lund, J. (1997), Stochastic Volatility and Mean Drift in the Short Rate Diffusion: Sources of Steepness, Level and Curvature in the Yield Curve, Working paper, Northwestern University.

Bekaert, G., Hodrick, R. J. and Marshall, D. A. (1997), 'On Biases in Tests of the Expectations Hypothesis of the Term Structure of Interest Rates', Journal of Financial Economics 44, 309-348.

Bollerslev, T. (1990), 'Modelling the Coherence in Short-Run Nominal Exchange Rates', Review of Economics and Statistics 72, 498-505.

Bollerslev, T. and Wooldridge, J. M. (1992), 'Quasimaximum Likelihood Estimation Dynamic Models with Time Varying Covariances', Econometric Reviews 11, 143-172.

Brown, R. H. and Schaefer, S. M. (1994), 'Interest Rate Volatility and the Shape of the Term Structure', Philosophical Transactions of the Royal Society A 347, 563-576.

Brown, R. H. and Schaefer, S. M. (2000), Why Long Term Forward Interest Rates (Almost) Always Slope Downwards, Working paper, London Business School.

Campbell, J. Y. and Shiller, R. J. (1991), 'Yield Spreads and Interest Rate Movements: A Bird's Eye View', Review of Economic Studies 58, 495-514.

Chen, L. (1996), Interest Rate Dynamics, Derivatives Pricing, and Risk Management, Springer Verlag, Berlin.

Chen, R. and Scott, L. (1993), 'Maximum Likelihood Estimation for a Multifactor Equilibrium Model of the Term Structure of Interest Rates', Journal of Fixed Income $\mathbf{3}(3), 14-31$.

Collin-Dufresne, P. and Goldstein, R. S. (2002), 'Do Bonds Span the Fixed Income Markets? Theory and Evidence for Unspanned Stochastic Volatility', Journal of Finance (forthcoming).

Cox, J. C., Ingersoll, J. E. and Ross, S. A. (1985), 'A Theory of the Term Structure of Interest Rates', Econometrica 53, 385-408.

Dai, Q. and Singleton, K. J. (2000), 'Specification Analysis of Affine Term Structure Models', Journal of Finance 55(5), 1943-1978. 
Dai, Q. and Singleton, K. J. (2002), 'Expectations Puzzles, Time-varying Risk Premia, and Dynamic Models of the Term Structure', Journal of Financial Economics 63, 350 .

De Jong, F. (2000), 'Time Series and Cross-Section Information in Affine Term-Structure Models', Journal of Business \& Economic Statistics 18(3), 300-314.

Duffee, G. R. (1996), 'Idiosyncratic Variation of Treasury Bill Yields', Journal of Finance $\mathbf{5 1}(2), 527-551$.

Engle, R. F. and Ng, V. K. (1993), 'Time-Varying Volatility and the Dynamic Behavior of the Term Structure', Journal of Money, Credit and Banking 25(3, Part 1), 336-349.

Engle, R., Ng, V. K. and Rothschild, M. (1990), 'Asset Pricing with a Factor-ARCH Covariance Structure', Journal of Econometrics 45, 213-237.

Fama, E. F. and Bliss, R. R. (1987), 'The Information in Long-Maturity Forward Rates', American Economic Review 77, 680-692.

Fong, H. and Vasicek, O. (1991), 'Fixed-Income Volatility Management', Journal of Portfolio Management 17(4), 41-46.

Heath, D., Jarrow, R. and Morton, A. (1992), 'Bond Pricing and the Term Structure of Interest Rates: A New Methodology for Contingent Claims Valuation', Econometrica $60,77-105$.

Jones, F. J. (1991), 'Yield curve strategies', Yield Curve Strategies 1(September), 43-51.

Litterman, R. and Scheinkman, J. (1991), 'Common Factors Affecting the Bond Returns', Journal of Fixed Income 1, 54-61.

Litterman, R., Scheinkman, J. and Weiss, L. (1991), 'Volatility and the Yield Curve', Journal of Fixed Income 1(June), 49-53.

Longstaff, F. and Schwartz, E. (1992), 'Interest Rate Volatility and the Term Structure: A Two-Factor General Equilibrium Model', Journal of Finance 47, 1259-1282. 


\begin{tabular}{l|l|ccccccc} 
& & 2 & 3 & 5 & 7 & 10 & 15 \\
& & month & year & year & year & year & year & year \\
\hline 1970-98 & Mean & 6.85 & 7.58 & 7.76 & 8.00 & 8.14 & 8.28 & 8.40 \\
& Std. dev. & 0.28 & 0.21 & 0.20 & 0.18 & 0.17 & 0.17 & 0.17 \\
& Skewness & -0.50 & -0.61 & -0.56 & -0.53 & -0.50 & -0.37 & -0.15 \\
& Kurtosis & 18.12 & 11.36 & 9.87 & 9.01 & 8.67 & 8.34 & 10.56 \\
& AC, 1 & 0.994 & 0.996 & 0.996 & 0.996 & 0.996 & 0.996 & 0.996 \\
& AC, 5 & 0.950 & 0.969 & 0.971 & 0.975 & 0.975 & 0.977 & 0.978 \\
& AC, sq, 1 & 0.241 & 0.228 & 0.195 & 0.198 & 0.207 & 0.201 & 0.268 \\
& AC, sq, 5 & 0.186 & 0.124 & 0.116 & 0.172 & 0.221 & 0.243 & 0.166 \\
\hline 1970-78 & Mean & 5.98 & 6.65 & 6.81 & 6.99 & 7.10 & 7.18 & 7.26 \\
& Std. dev. & 0.22 & 0.17 & 0.15 & 0.13 & 0.11 & 0.12 & 0.14 \\
& Skewness & -0.29 & -0.21 & -0.33 & -0.53 & -0.46 & -0.00 & 0.50 \\
& Kurtosis & 9.01 & 5.19 & 5.63 & 6.41 & 6.00 & 9.67 & 26.68 \\
& AC, 1 & 0.981 & 0.972 & 0.977 & 0.979 & 0.980 & 0.979 & 0.971 \\
& AC, 5 & 0.888 & 0.865 & 0.866 & 0.877 & 0.888 & 0.897 & 0.893 \\
& AC, sq, 1 & 0.131 & 0.032 & 0.038 & 0.055 & 0.087 & 0.365 & 0.462 \\
& AC, sq, 5 & 0.010 & -0.002 & 0.003 & 0.024 & 0.051 & 0.033 & 0.001 \\
\hline Mean & 11.83 & 11.79 & 11.66 & 11.54 & 11.49 & 11.46 & 11.46 \\
& Std. dev. & 0.63 & 0.41 & 0.37 & 0.33 & 0.31 & 0.30 & 0.31 \\
& Skewness & -0.21 & -0.55 & -0.59 & -0.65 & -0.65 & -0.57 & -0.42 \\
& Kurtosis & 4.95 & 5.09 & 5.11 & 5.05 & 4.91 & 4.60 & 4.33 \\
& AC, 1 & 0.966 & 0.975 & 0.978 & 0.980 & 0.981 & 0.981 & 0.980 \\
& AC, 5 & 0.754 & 0.809 & 0.842 & 0.870 & 0.882 & 0.892 & 0.903 \\
& AC sq, 1 & 0.107 & 0.102 & 0.099 & 0.106 & 0.114 & 0.073 & 0.016 \\
& AC, sq, 5 & 0.029 & 0.000 & 0.016 & 0.087 & 0.148 & 0.184 & 0.162 \\
\hline Mean & 6.09 & 7.05 & 7.32 & 7.67 & 7.89 & 8.10 & 8.28 \\
& Std. dev. & 0.14 & 0.15 & 0.16 & 0.16 & 0.15 & 0.15 & 0.14 \\
& Skewness & -1.74 & -0.17 & -0.07 & 0.01 & 0.04 & 0.05 & 0.14 \\
& Kurtosis & 20.68 & 5.06 & 4.63 & 4.32 & 4.21 & 4.23 & 4.42 \\
& AC, 1 & 0.996 & 0.995 & 0.995 & 0.995 & 0.994 & 0.994 & 0.995 \\
& AC, 5 & 0.990 & 0.972 & 0.971 & 0.970 & 0.970 & 0.970 & 0.970 \\
AC, sq, 1 & 0.168 & 0.130 & 0.131 & 0.128 & 0.122 & 0.123 & 0.129 \\
& AC, sq, 5 & 0.005 & 0.027 & 0.017 & 0.007 & 0.004 & 0.012 & 0.033
\end{tabular}

The table provides summary statistics for yields (in \%) for selected maturities. The mean, and autocorrelation are calculated for the yields in levels. The standard deviation, skewness, kurtosis, and the autocorrelation of the squared time series are calculated for the yields in first differences. The weekly data cover the periods 1970-98, 1970-78, 1979-82, and 1983-98.

Table 1: Yield Summary Statistics 


\begin{tabular}{l|l|ccccccc} 
& & Mean & Std. dev. & Pct. neg. & AC, 1 & AC, sq, 1 & Corr $s_{t}$ & Corr $c_{t}$ \\
\hline $1970-98$ & $\Delta l_{t}$ & 0.002 & 0.283 & 50.4 & 0.180 & 0.241 & -0.078 & -0.083 \\
& $s_{t}$ & 1.429 & 1.513 & 15.2 & 0.991 & 0.944 & & 0.878 \\
& $c_{t}$ & 0.510 & 0.642 & 16.6 & 0.972 & 0.916 & & \\
\hline $1970-78$ & $\Delta l_{t}$ & 0.002 & 0.220 & 48.8 & 0.206 & 0.133 & -0.100 & -0.079 \\
& $s_{t}$ & 1.204 & 1.195 & 18.2 & 0.981 & 0.935 & & 0.914 \\
& $c_{t}$ & 0.486 & 0.538 & 17.3 & 0.964 & 0.922 & & \\
\hline $1979-82$ & $\Delta l_{t}$ & -0.006 & 0.628 & 48.3 & 0.204 & 0.109 & -0.144 & -0.140 \\
& $s_{t}$ & -0.368 & 2.006 & 61.2 & 0.965 & 0.881 & & 0.957 \\
& $c_{t}$ & -0.059 & 1.097 & 57.4 & 0.959 & 0.896 & & \\
\hline $1983-98$ & $\Delta l_{t}$ & -0.004 & 0.140 & 51.7 & 0.020 & 0.168 & -0.006 & 0.025 \\
& $s_{t}$ & 2.003 & 1.084 & 2.0 & 0.991 & 0.972 & & 0.745 \\
& $c_{t}$ & 0.665 & 0.423 & 6.1 & 0.975 & 0.910 & &
\end{tabular}

The table provides summary statistics for $\Delta$ level, slopes and butterfly spreads calculated in the following manner: $l_{t}=y_{1 t}, s_{t}=y_{3 t}-y_{1 t}$ and $c_{t}=y_{2 t}-\left(w y_{1 t}+(1-w) y_{3 t}\right)$ where $y_{1}$ is the 3 month yield, $y_{2}$ is the 3 year yield, and $y_{3}$ is the 10 year yield. $w$ is the weight such that the duration of the long and short part of the butterfly spread are equal. The weekly data cover the periods $1970-98,1970-78,1979-82$, and $1983-98$

Table 2: Slope and Butterfly Summary Statistics 
Panel A: 1970-98

\begin{tabular}{c|cccccc} 
& $l_{t}$ & \multicolumn{3}{c}{$s_{t}$} & & \\
\hline$\gamma$ & -0.041 & $(0.063)$ & 0.062 & $(0.057)$ & 0.053 & $(0.039)$ \\
\hline$\omega$ & $0.000^{*}$ & $(0.000)$ & $0.001^{*}$ & $(0.000)$ & $0.000 \ddagger$ & $(0.000)$ \\
$\alpha$ & $0.120 \ddagger$ & $(0.043)$ & $0.070^{*}$ & $(0.022)$ & $0.075^{*}$ & $(0.023)$ \\
$\beta$ & $0.869^{*}$ & $(0.035)$ & $0.887^{*}$ & $(0.031)$ & $0.898^{*}$ & $(0.031)$
\end{tabular}

Panel B: $1970-78$

\begin{tabular}{c|cccccc}
\hline$\gamma$ & $-0.439 \ddagger$ & $(0.243)$ & 0.329 & $(0.221)$ & $0.208 \dagger$ & $(0.127)$ \\
\hline$\omega$ & 0.001 & $(0.000)$ & 0.001 & $(0.001)$ & 0.000 & $(0.000)$ \\
$\alpha$ & $0.061 \ddagger$ & $(0.032)$ & 0.050 & $(0.035)$ & $0.085 \dagger$ & $(0.044)$ \\
$\beta$ & $0.920^{*}$ & $(0.033)$ & $0.920^{*}$ & $(0.053)$ & $0.884^{*}$ & $(0.064)$
\end{tabular}

Panel C: $1979-82$

\begin{tabular}{c|cccccc}
\hline$\gamma$ & -0.314 & $(0.408)$ & 0.163 & $(0.239)$ & 0.056 & $(0.127)$ \\
\hline$\omega$ & 0.010 & $(0.010)$ & 0.005 & $(0.005)$ & 0.001 & $(0.001)$ \\
$\alpha$ & 0.088 & $(0.086)$ & 0.083 & $(0.087)$ & 0.078 & $(0.054)$ \\
$\beta$ & $0.894^{*}$ & $(0.099)$ & $0.903^{*}$ & $(0.090)$ & $0.922^{*}$ & $(0.054)$
\end{tabular}

Panel D: $1983-98$

\begin{tabular}{c|cccccc}
\hline$\gamma$ & -0.065 & $(0.095)$ & $0.206 \ddagger$ & $(0.102)$ & $0.236^{*}$ & $(0.074)$ \\
\hline$\omega$ & $0.001 \ddagger$ & $(0.000)$ & $0.001 \ddagger$ & $(0.001)$ & $0.001^{*}$ & $(0.000)$ \\
$\alpha$ & $0.217^{*}$ & $(0.071)$ & $0.048^{*}$ & $(0.016)$ & $0.076^{*}$ & $(0.022)$ \\
$\beta$ & $0.764^{*}$ & $(0.058)$ & $0.872^{*}$ & $(0.044)$ & $0.800^{*}$ & $(0.039)$
\end{tabular}

QML estimates of the VAR(4)-CCORR-M model for $x_{t}^{\prime} \equiv\left(\Delta l_{t} s_{t} c_{t}\right), l_{t}=y_{1 t}, s_{t}=y_{3 t}-y_{1 t}$ and $c_{t}=y_{2 t}-\left(w y_{1 t}+(1-w) y_{3 t}\right)$ where $y_{1}$ is the 3 month yield, $y_{2}$ is the 3 year yield, $y_{3}$ is the 10 year yield, and $w=\frac{28}{39}$. $x_{t}=\Phi_{0}+\Phi_{1} x_{t-1}+\Phi_{2} x_{t-2}+\Phi_{3} x_{t-3}+\Phi_{4} x_{t-4}+\gamma \sqrt{h_{1 t}}+\varepsilon_{t}$. $\varepsilon_{t}$ has mean 0 and conditional covariance $H_{t}: h_{i t}=\omega_{i}+\alpha_{i} \varepsilon_{i t-1}^{2}+\beta_{i} h_{i t-1}$, for $i=1,2,3$ and $h_{i j t}=\rho_{i j} \sqrt{h_{i t} h_{j t}}$ for $i, j=1,2,3$ and $i \neq j . \Phi_{0}, \Phi_{1}, \Phi_{2}, \Phi_{3}, \Phi_{4}$ not reported. The weekly data cover the periods $1970-98$, 1970-78, 1979-82, and 1983-98. Bollerslev and Wooldridge (1992) robust standard errors in parenthesis. $\dagger(\ddagger)[*]$ indicates that the parameter is significantly different from zero at a $10 \%(5 \%)[1 \%]$ level.

Table 3: VAR-CCORR-M Model; Various Periods 
Panel A: 3 months $/ 3$ years $/ 5$ years

\begin{tabular}{c|cccccc} 
& $l_{t}$ & \multicolumn{3}{c}{$s_{t}$} & \\
\hline$\gamma$ & -0.090 & $(0.091)$ & $0.247^{*}$ & $(0.093)$ & $0.119^{*}$ & $(0.039)$ \\
\hline$\omega$ & $0.001 \dagger$ & $(0.000)$ & $0.002^{*}$ & $(0.001)$ & $0.000^{*}$ & $(0.000)$ \\
$\alpha$ & $0.230^{*}$ & $(0.084)$ & $0.038 \ddagger$ & $(0.015)$ & $0.064^{*}$ & $(0.023)$ \\
$\beta$ & $0.753^{*}$ & $(0.072)$ & $0.866^{*}$ & $(0.061)$ & $0.773^{*}$ & $(0.068)$
\end{tabular}

Panel B: 3 months $/ 3$ years $/ 7$ years

\begin{tabular}{c|cccccc}
\hline$\gamma$ & -0.078 & $(0.093)$ & $0.233 \ddagger$ & $(0.098)$ & $0.186^{*}$ & $(0.059)$ \\
\hline$\omega$ & $0.001 \ddagger$ & $(0.000)$ & $0.001 \ddagger$ & $(0.001)$ & $0.001^{*}$ & $(0.000)$ \\
$\alpha$ & $0.223^{*}$ & $(0.076)$ & $0.039^{*}$ & $(0.014)$ & $0.068^{*}$ & $(0.022)$ \\
$\beta$ & $0.758^{*}$ & $(0.064)$ & $0.878^{*}$ & $(0.046)$ & $0.797^{*}$ & $(0.045)$
\end{tabular}

Panel C: 3 months $/ 3$ years $/ 10$ years

\begin{tabular}{c|cccccc}
\hline$\gamma$ & -0.065 & $(0.095)$ & $0.206_{\ddagger}^{\ddagger}$ & $(0.102)$ & $0.236^{*}$ & $(0.074)$ \\
\hline$\omega$ & $0.001 \ddagger$ & $(0.000)$ & $0.001^{\ddagger}$ & $(0.001)$ & $0.001^{*}$ & $(0.000)$ \\
$\alpha$ & $0.217^{*}$ & $(0.071)$ & $0.048^{*}$ & $(0.016)$ & $0.076^{*}$ & $(0.022)$ \\
$\beta$ & $0.764^{*}$ & $(0.058)$ & $0.872^{*}$ & $(0.044)$ & $0.800^{*}$ & $(0.039)$
\end{tabular}

Panel D: 3 months $/ 3$ years $/ 15$ years

\begin{tabular}{c|cccccc}
\hline$\gamma$ & -0.056 & $(0.096)$ & $0.178 \dagger$ & $(0.106)$ & $0.270^{*}$ & $(0.082)$ \\
\hline$\omega$ & $0.001 \ddagger$ & $(0.000)$ & $0.001^{\ddagger}$ & $(0.000)$ & $0.001^{*}$ & $(0.000)$ \\
$\alpha$ & $0.214^{*}$ & $(0.069)$ & $0.056^{*}$ & $(0.019)$ & $0.080^{*}$ & $(0.022)$ \\
$\beta$ & $0.768^{*}$ & $(0.056)$ & $0.875^{*}$ & $(0.040)$ & $0.802^{*}$ & $(0.034)$
\end{tabular}

QML estimates of the VAR(4)-CCORR-M model for $x_{t}^{\prime} \equiv\left(\Delta l_{t} s_{t} c_{t}\right), l_{t}=y_{1 t}, s_{t}=y_{3 t}-y_{1 t}$ and $c_{t}=y_{2 t}-\left(w y_{1 t}+(1-w) y_{3 t}\right)$ where, $y_{1}$ is the 3 month yield, $y_{2}$ is the 3 year yield, and $y_{3}$ is the 5 year, 7 year, 10 year, and 15 year yield, respectively. $x_{t}=\Phi_{0}+\Phi_{1} x_{t-1}+\Phi_{2} x_{t-2}+\Phi_{3} x_{t-3}+\Phi_{4} x_{t-4}+\gamma \sqrt{h_{1 t}}+\varepsilon_{t}$. $\varepsilon_{t}$ has mean 0 and conditional covariance $H_{t}: h_{i t}=\omega_{i}+\alpha_{i} \varepsilon_{i t-1}^{2}+\beta_{i} h_{i t-1}$, for $i=1,2,3$, and $h_{i j t}=\rho_{i j} \sqrt{h_{i t} h_{j t}}$ for $i, j=1,2,3$ and $i \neq j . \Phi_{0}, \Phi_{1}, \Phi_{2}, \Phi_{3}, \Phi_{4}$ not reported. The weekly data cover the period 1983-98. Bollerslev and Wooldridge (1992) robust standard errors in parenthesis. $\dagger(\ddagger)[*]$ indicates that the parameter is significantly different from zero at a $10 \%(5 \%)[1 \%]$ level.

Table 4: VAR-CCORR-M Model; Various Long Maturities 
Panel A: 3 months/2 years $/ 10$ years

\begin{tabular}{c|cccccc} 
& $l_{t}$ & \multicolumn{3}{c}{$s_{t}$} & \\
\hline$\gamma$ & -0.093 & $(0.092)$ & $0.221_{t}^{\ddagger}$ & $(0.096)$ & $0.226^{*}$ & $(0.074)$ \\
\hline$\omega$ & $0.001^{\ddagger}$ & $(0.000)$ & $0.001 \ddagger$ & $(0.001)$ & $0.001^{*}$ & $(0.001)$ \\
$\alpha$ & $0.219^{*}$ & $(0.075)$ & $0.047^{*}$ & $(0.015)$ & $0.076^{*}$ & $(0.024)$ \\
$\beta$ & $0.762^{*}$ & $(0.063)$ & $0.871^{*}$ & $(0.043)$ & $0.770^{*}$ & $(0.053)$
\end{tabular}

Panel B: 3 months $/ 3$ years $/ 10$ years

\begin{tabular}{c|cccccc}
\hline$\gamma$ & -0.065 & $(0.095)$ & $0.206_{\ddagger}^{\ddagger}$ & $(0.102)$ & $0.236^{*}$ & $(0.074)$ \\
\hline$\omega$ & $0.001^{\ddagger}$ & $(0.000)$ & $0.001 \ddagger$ & $(0.001)$ & $0.001^{*}$ & $(0.000)$ \\
$\alpha$ & $0.217^{*}$ & $(0.071)$ & $0.048^{*}$ & $(0.016)$ & $0.076^{*}$ & $(0.022)$ \\
$\beta$ & $0.764^{*}$ & $(0.058)$ & $0.872^{*}$ & $(0.044)$ & $0.800^{*}$ & $(0.039)$
\end{tabular}

Panel C: 3 months $/ 5$ years $/ 10$ years

\begin{tabular}{c|cccccc}
\hline$\gamma$ & -0.015 & $(0.100)$ & 0.168 & $(0.108)$ & $0.168^{*}$ & $(0.060)$ \\
\hline$\omega$ & $0.001 \ddagger$ & $(0.000)$ & $0.001 \ddagger$ & $(0.001)$ & $0.000^{*}$ & $(0.000)$ \\
$\alpha$ & $0.208^{*}$ & $(0.065)$ & $0.057^{*}$ & $(0.019)$ & $0.087^{*}$ & $(0.022)$ \\
$\beta$ & $0.777^{*}$ & $(0.051)$ & $0.860^{*}$ & $(0.048)$ & $0.804^{*}$ & $(0.036)$
\end{tabular}

Panel D: 3 months $/ 7$ years $/ 10$ years

\begin{tabular}{c|cccccc}
\hline$\gamma$ & 0.017 & $(0.117)$ & 0.152 & $(0.114)$ & $0.093 \ddagger$ & $(0.037)$ \\
\hline$\omega$ & $0.001 \ddagger$ & $(0.000)$ & $0.001 \ddagger$ & $(0.000)$ & 0.000 & $(0.000)$ \\
$\alpha$ & $0.205^{*}$ & $(0.065)$ & $0.063^{*}$ & $(0.021)$ & $0.086^{*}$ & $(0.023)$ \\
$\beta$ & $0.783^{*}$ & $(0.052)$ & $0.849^{*}$ & $(0.054)$ & $0.798^{*}$ & $(0.041)$
\end{tabular}

QML estimates of the VAR(4)-CCORR-M model for $x_{t}^{\prime} \equiv\left(\Delta l_{t} s_{t} c_{t}\right), l_{t}=y_{1 t}, s_{t}=y_{3 t}-y_{1 t}$ and $c_{t}=y_{2 t}-\left(w y_{1 t}+(1-w) y_{3 t}\right)$ where, $y_{1}$ is the 3 month yield, $y_{2}$ is the 2 year, 3 year, 5 year, and 7 year yield, respectively, and $y_{3}$ is the 10 year yield. $x_{t}=\Phi_{0}+\Phi_{1} x_{t-1}+\Phi_{2} x_{t-2}+\Phi_{3} x_{t-3}+\Phi_{4} x_{t-4}+\gamma \sqrt{h_{1 t}}+\varepsilon_{t}$. $\varepsilon_{t}$ has mean 0 and conditional covariance $H_{t}: h_{i t}=\omega_{i}+\alpha_{i} \varepsilon_{i t-1}^{2}+\beta_{i} h_{i t-1}$, for $i=1,2,3$, and $h_{i j t}=\rho_{i j} \sqrt{h_{i t} h_{j t}}$ for $i, j=1,2,3$ and $i \neq j . \Phi_{0}, \Phi_{1}, \Phi_{2}, \Phi_{3}, \Phi_{4}$ not reported. The weekly data cover the period 1983-98. Bollerslev and Wooldridge (1992) robust standard errors in parenthesis. $\dagger(\ddagger)[*]$ indicates that the parameter is significantly different from zero at a $10 \%(5 \%)[1 \%]$ level.

Table 5: VAR-CCORR-M Model; Various Medium Maturities 


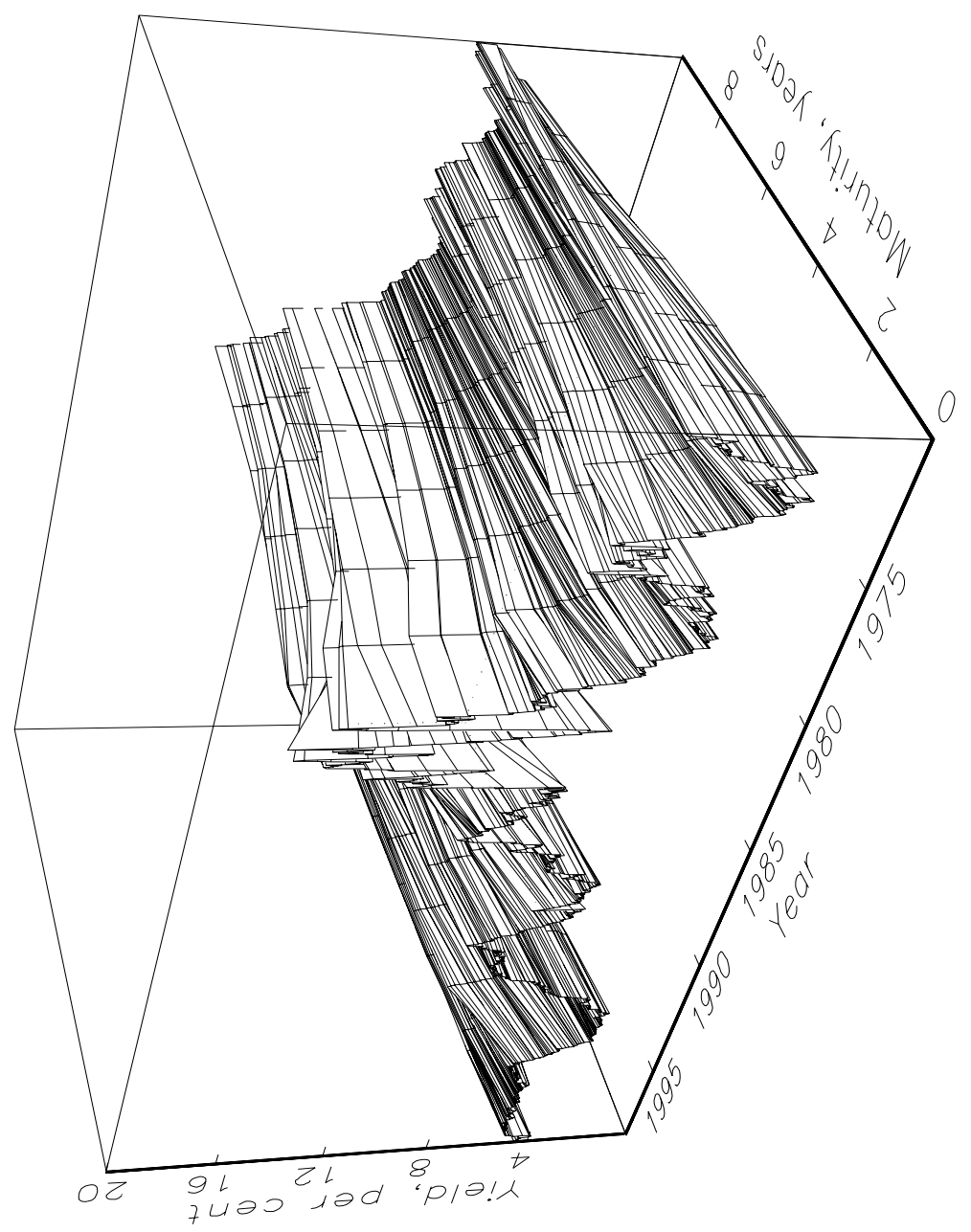

Figure 1: Yield Curve Surface Plot 


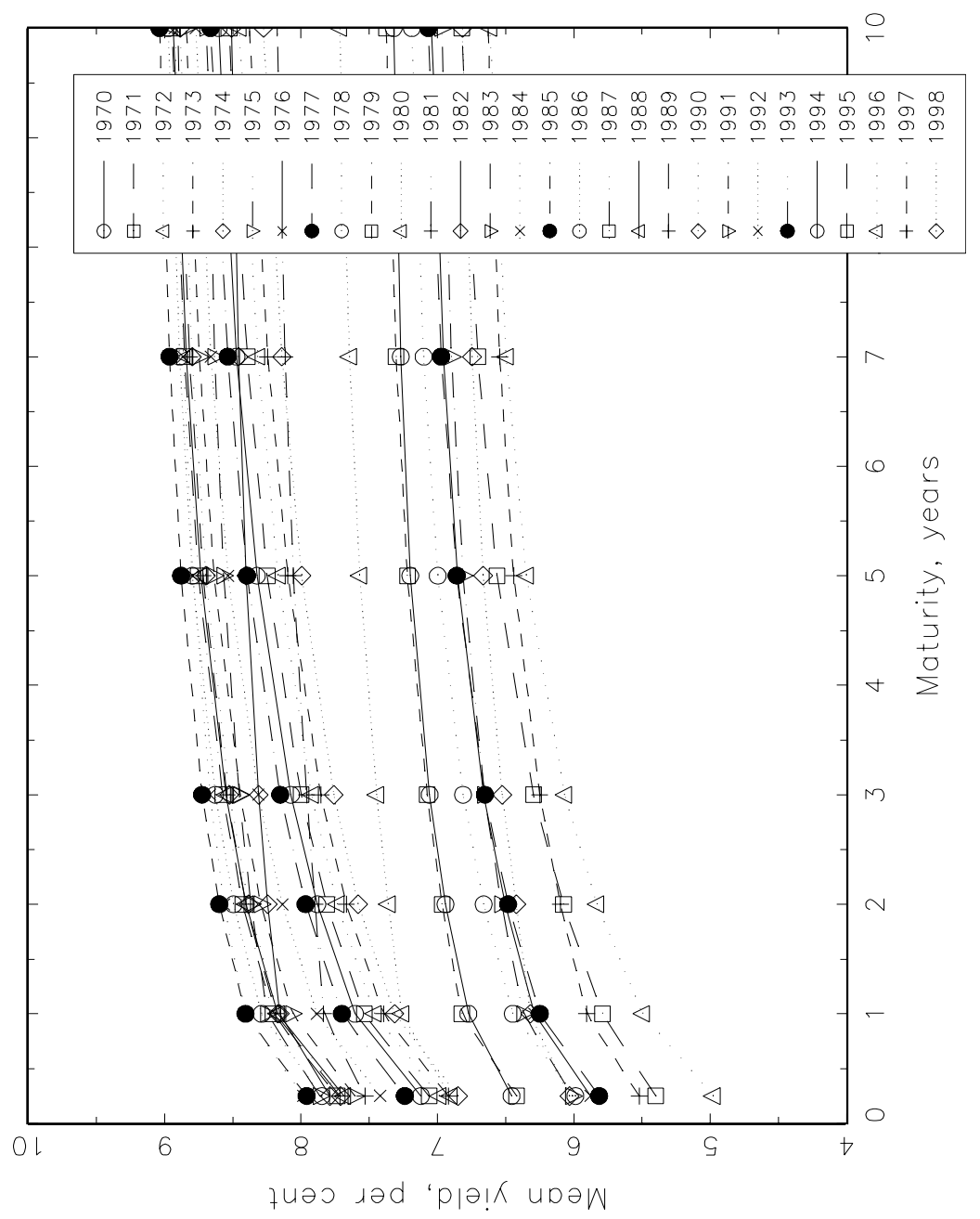

Figure 2: Average Yield Curves 


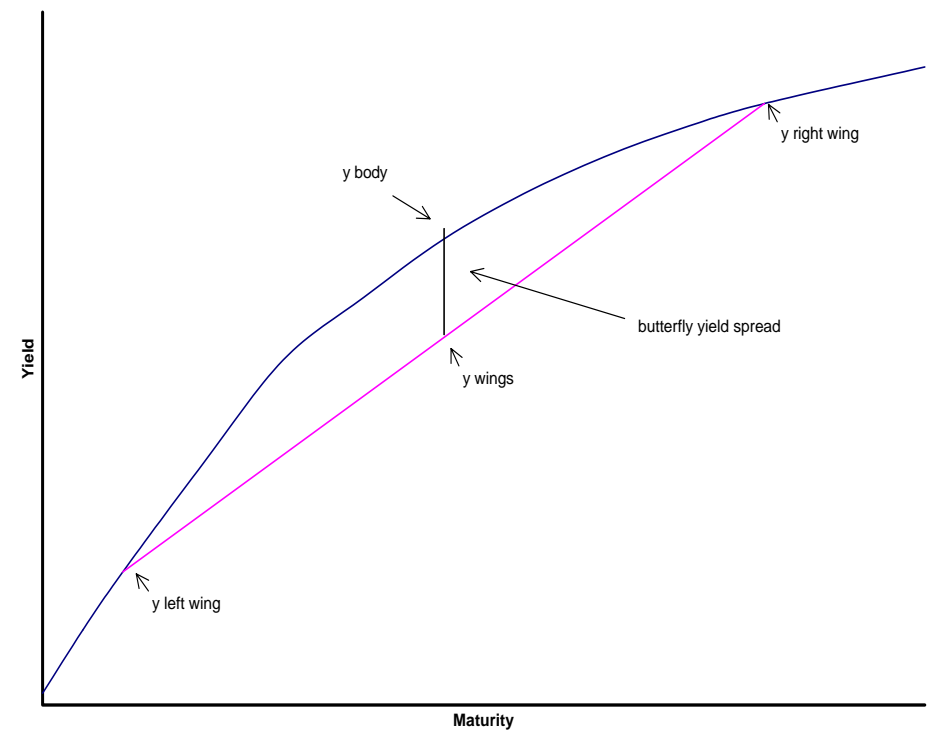

Figure 3: Butterfly Yield Spread 


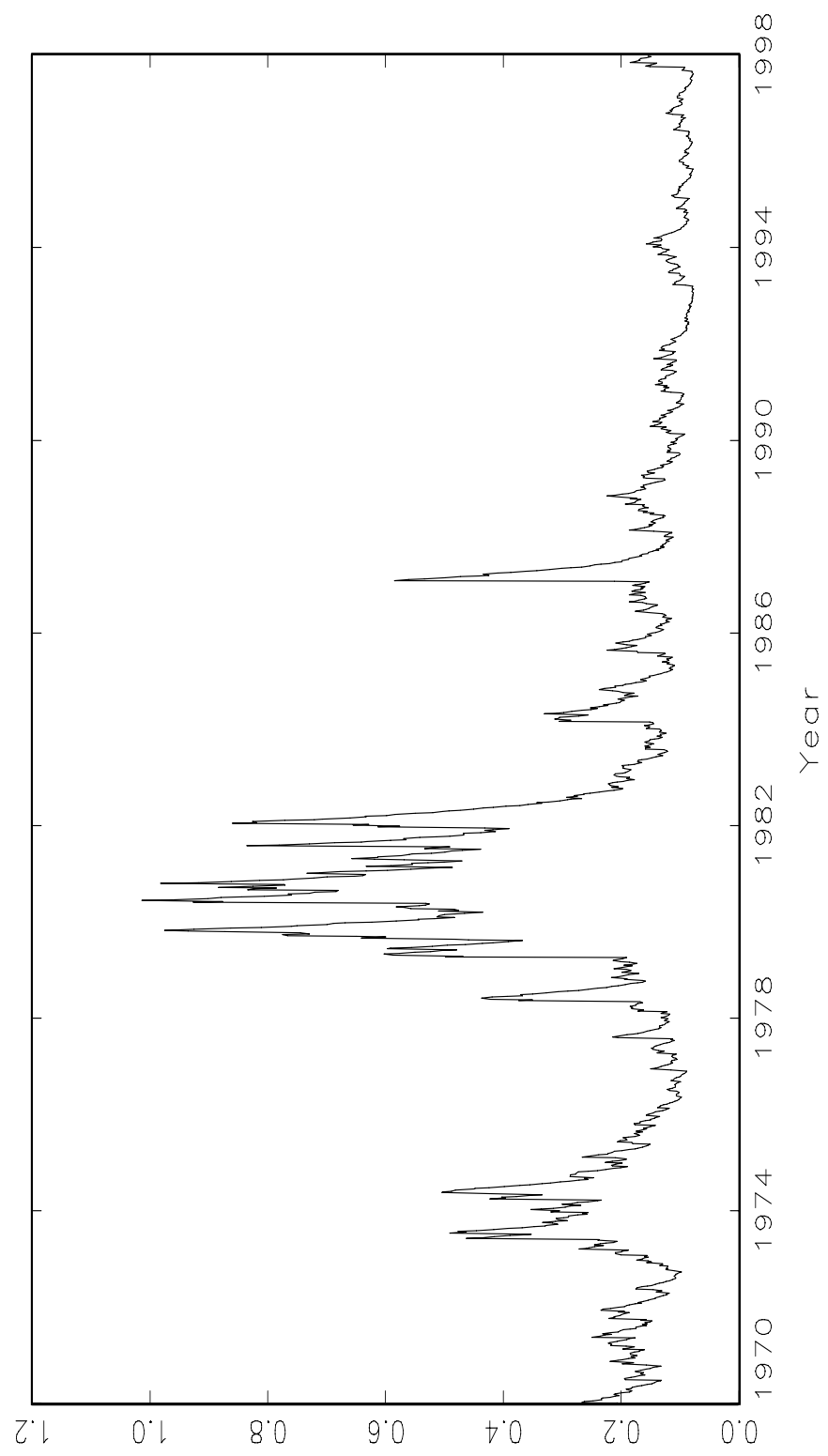

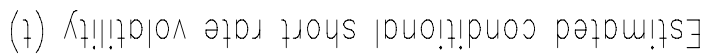

Figure 4: Estimated Volatility of Short-Rate Changes: 1970-98 